\title{
Magnetic properties and neutron diffraction of $\mathrm{TbMn}_{4} \mathrm{Al}_{8}$
}

\author{
P. Schobinger-Papamantellos ${ }^{\mathrm{a}}$, P. Fischer ${ }^{\mathrm{b}}$, C.H. de Groot ${ }^{\mathrm{c}}$, F.R. de Boer ${ }^{\mathrm{c}}$, K.H.J. Buschow ${ }^{\mathrm{c}}$ \\ ${ }^{2}$ Laboratorium für Kristallographie ETH Zürich, $\mathrm{CH}-8092$ Zürich, Switzerland \\ ${ }^{\circ}$ Laboratory for Neutron Scattering ETHZ \& PSI, CH-5232, Villingen, Switzerland \\ 'Van der Waals-Zeeman Laboratory, University of Amsterdam Valckenierstr. 65, 1018 XE Amsterdam, Netheriands
}

Received 9 May 1995

\begin{abstract}
We have studied the magnetic properties of the compound $\mathrm{TbMn}_{4} \mathrm{Al}_{8}$ by means of neutron diffraction and magnetic measurements. From the magnetic measurements it is concluded that the magnetic interactions in this compound are very weak and do not lead to magnetic ordering above $4.2 \mathrm{~K}$. From the neutron diffraction measurements it is concluded that magnetic ordering is absent even down to $1.5 \mathrm{~K}$. The influence of atomic-site disorder on the magnetic properties is discussed.
\end{abstract}

Keywords: $\mathrm{TbMn}_{4} \mathrm{Al}_{8} ;$ Magnetic properties; Neutron diffraction

\section{Introduction}

The tetragonal $\mathrm{ThMn}_{12}$-type structure is a comparatively simple crystal structure characterised by a single Th position and three Mn positions. The latter three positions differ markedly regarding their co-ordination. Numerous ternary rare-earth $(\mathrm{R})$ compounds $\left(\mathrm{RT}_{12-x} \mathrm{M}_{x}\right)$ form in this structure type; the $3 \mathrm{~d}$-transition-metal atoms $\mathrm{T}$ and the s-, p-element atoms $\mathrm{M}$ show a strong preference for one or two of the three positions mentioned [1-4]. The magnetic properties of the compounds based on $\mathrm{Fe}, \mathrm{RFe}_{4} \mathrm{Al}_{8}$, are dominated by the $\mathrm{Fe}$ sublattice that gives rise to antiferromagnetic ordering at a temperature of about 170 $\mathrm{K}[2]$. The rare-earth moments order at much lower temperatures owing to the relatively weak magnetic coupling between the Fe sublattice and the $\mathrm{R}$ sublattice.

The situation is less clear for the $\mathrm{Mn}$ compounds, $\mathrm{RMn}_{4} \mathrm{Al}_{8}$. No magnetic ordering and no magnetic moments on the $\mathrm{Mn}$ atoms above $4.2 \mathrm{~K}$ were found in a previous investigation [2] of the compounds $\mathrm{GdMn} \mathrm{Al}_{8}$ and $\mathrm{YMn}_{4} \mathrm{Al}_{8}$, suggesting that the situation is not much different in the other $\mathrm{RMn}_{4} \mathrm{Al}_{8}$ compounds. By contrast, magnetic ordering and $\mathrm{Mn}$ moments in excess of $1 \mu_{\mathrm{B}}$ in several $\mathrm{RMn}_{4} \mathrm{Al}_{8}$ compounds were reported in Ref. [3]. More recently, Coldea and co-workers [5,6] analysed their high temperature data of $\mathrm{GdMn}_{4} \mathrm{Al}_{8}$ and $\mathrm{YMn}_{4} \mathrm{Al}_{8}$ in terms of spin fluctuations. These authors propose that below $300 \mathrm{~K}$ only a few percent of the Mn atoms carry a moment and that this property is closely associated with the crystallographic site which the $\mathrm{Mn}$ atoms occupy. In order to clarify the situation regarding the magnetism in the $\mathrm{RMn}_{4} \mathrm{Al}_{8}$, we have investigated the compound $\mathrm{TbMn}_{4} \mathrm{Al}_{8}$ by means of neutron diffraction and magnetic measurements.

\section{Experimental}

The sample of the compound $\mathrm{TbMn}_{4} \mathrm{Al}_{8}$ was prepared starting from materials of at least $99.9 \%$ purity by standard arc-melting. Subsequently, the sample was wrapped in Ta-foil and inserted into a quartz tube in which it was vacuum annealed for 2 weeks at $800^{\circ} \mathrm{C}$. Examination of the annealed sample by X-ray diffraction showed that it was single phase. All diffraction lines were indexed according to the tetragonal $\mathrm{ThMn}_{12}$-structure type.

The magnetic measurements were made on powder samples by means of a SQUID magnetometer in the temperature range $4.2-300 \mathrm{~K}$ in fields up to $5.5 \mathrm{~T}$.

Neutron diffraction measurements were made at the Saphir reactor in Wuerenlingen on powdered material at various temperatures (a) on the DMC (Double axis multicounter system) using $\lambda=0.1704 \mathrm{~nm}(6,26$ and $300 \mathrm{~K}$ ) and employing the high intensity mode and (b) 
in the double axis diffractometer P2ax, using $\lambda=$ $0.2337 \mathrm{~nm}(1.5$ and $8.0 \mathrm{~K})$ [7]. The step increment of the diffraction angle $2 \theta$ was $0.1^{\circ}$. The data were corrected for absorption and analysed by using the Rietveld line profile analysis method [8,9]. The scattering lengths were taken from the data listed in Ref. $[10]$.

\section{Experimental results and discussion}

Results of magnetic measurements made on the compound $\mathrm{TbMn}_{4} \mathrm{Al}_{8}$ are displayed in Figs. 1 and 2. It can be inferred from these results that the magnetic susceptibility follows Curie-Weiss behaviour over al-

$\mathrm{TbMn}_{4} \mathrm{Al}_{8}$

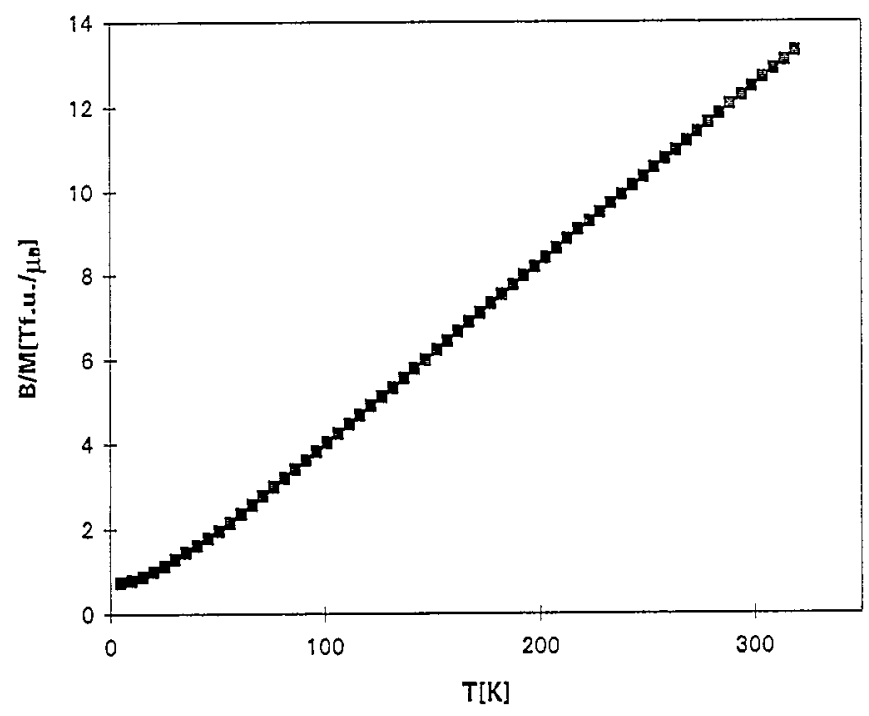

Fig. 1. Temperature dependence of the magnetic susceptibility in $\mathrm{TbMn}_{4} \mathrm{Al}_{8}$ derived from magnetisation measurements made in a field of $5 \mathrm{~T}$.

$\mathrm{TbMn}_{4} \mathrm{Al}$

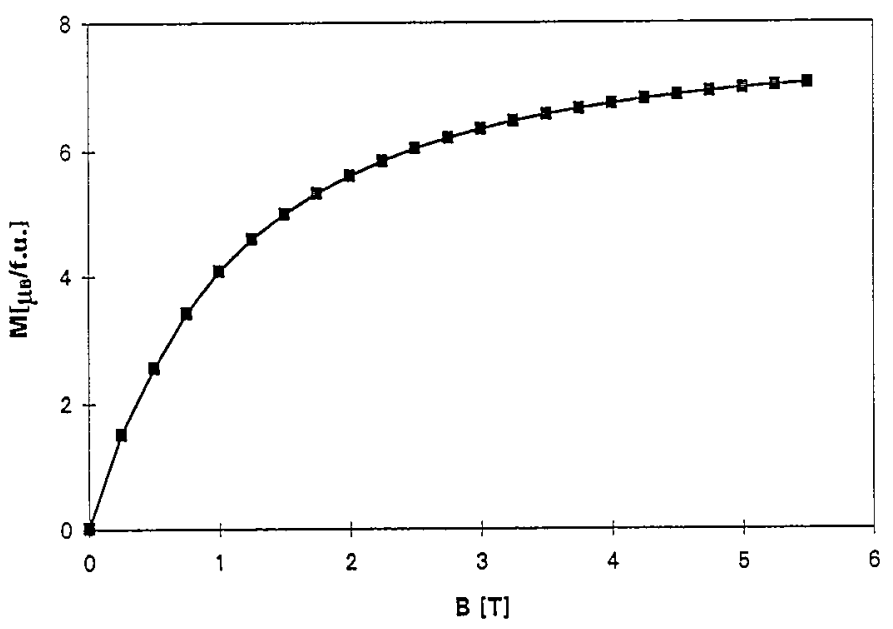

Fig. 2. Field dependence of the magnetisation of $\mathrm{TbMn}_{4} \mathrm{Al}_{8}$ at $4.2 \mathrm{~K}$. most the whole temperature range considered. The Curie-Weiss intercept $\theta_{\mathrm{p}}$ is close to zero and the effective moment derived from the slope of the plot is $\mu_{\text {eff }}=10.3 \mu_{\mathrm{B}}$ per formula unit $\mathrm{TbMn}_{4} \mathrm{Al}_{8}$. This value is close to the free-ion value $g[J(J+1)]^{1 / 2} \mu_{\mathrm{B}}=9.72$ $\mu_{\mathrm{B}}$. That the former value is slightly larger than the latter may be due to a small contribution from the $5 \mathrm{~d}$ valence electron polarisation of the $\mathrm{Tb}$ atoms. Alternatively, it may reflect the presence of a magnetic moment on a small fraction of the $\mathrm{Mn}$ atoms, as proposed by Coldea and co-workers [5,6]. The results presented in Fig. 1 do not give any indication of magnetic ordering occurring above $4.2 \mathrm{~K}$.

The field dependence of the magnetisation of $\mathrm{TbMn}_{4} \mathrm{Al}_{8}$ measured with decreasing field strengths at $4.2 \mathrm{~K}$ is shown in Fig. 2. This curve has a shape typical for a paramagnetic system. This result is consistent with the above conclusion that there is no magnetic ordering at $4.2 \mathrm{~K}$ or above.

The neutron diffraction diagrams of $\mathrm{TbMn}_{4} \mathrm{Al}_{8}$ taken at 295, 26 and $10 \mathrm{~K}$ are shown in Figs. 3 and 4. The latter two diagrams were collected with a 30 times higher counting rate than the room temperature diagram. Except for a small intensity contribution at $2 \theta \approx 27^{\circ}$, these diagrams do not contain any more peaks distinguishable from the background by more than $1 \%$ than the room temperature diagram. Several attempts to index this reflection by assuming a $P$ lattice or a magnetic cell that is a simple multiple of the nuclear cell could not explain this single observation. When assuming a wave vector $q=(0,0,0.78)$ along $c$ one may explain this observation as a satellite reflection of $002 \pm q_{z}$. However, a single observation does not provide sufficient experimental support for deriving a model since this tiny reflection could originate from a secondary phase. Further confirmation of the fact that magnetic ordering at $10 \mathrm{~K}$ is absent was obtained by inspection of the difference diagram based on the $10 \mathrm{~K}$ and $26 \mathrm{~K}$ data.

In passing, we note that our results are completely different from those obtained by neutron diffraction on the isomorphic compound $\mathrm{HoFe}_{4} \mathrm{Al}_{8}$. In the latter compound there is clear evidence of magnetic satellites on either side of the 200/101 and 220/211 reflections, which were interpreted as arising from incommensurate long range magnetic ordering of the $\mathrm{Fe}$ sublattice [11]. By contrast, our results do not provide evidence for long range magnetic ordering of the $\mathrm{Mn}$ sublattice, at least not for Mn moments larger than 0.2 $\mu_{\mathrm{B}}$ per atom.

Results of the refinement based on space group $14 / \mathrm{mmm}$ and on the presence of only nuclear reflections are presented in Table 1 . These results confirm the conclusion that there is a strong preference of the $\mathrm{Al}$ atoms for occupying the 8 (i) and 8(j) sites and of the $\mathrm{Mn}$ atoms for occupying the $8(\mathrm{f})$ site [4]. 
Table 1

Refined positional parameters and site occupancy of $\mathrm{TbMn}_{4} \mathrm{Al}_{8}(I 4 / \mathrm{mmm})$ at $295 \mathrm{~K}, 26 \mathrm{~K}$ and $10 \mathrm{~K} ; R_{\mathrm{n}}$, $R_{\text {ipp }}$ are the reliability factors for the integrated nuclear intensities and the weighted profile

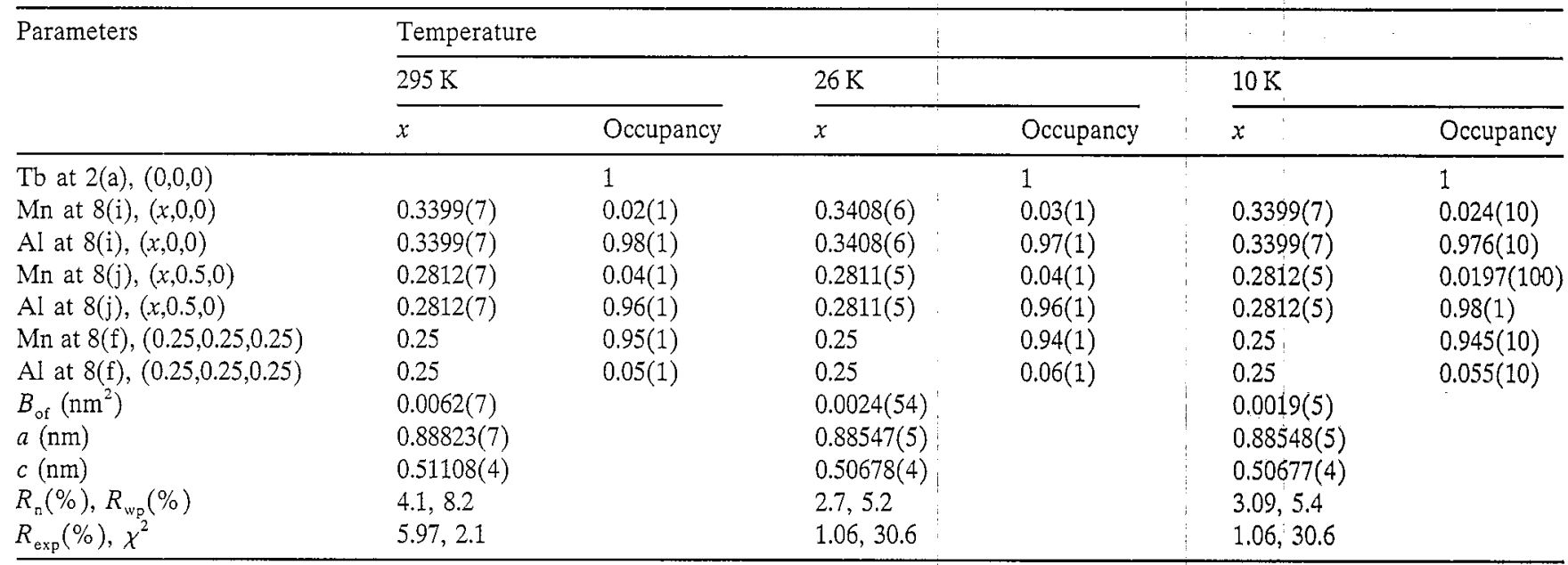

Neutron diffraction diagrams were also taken at 8.0 and $1.5 \mathrm{~K}$ in order to investigate whether the compound $\mathrm{TbMn}_{4} \mathrm{Al}_{8}$ is magnetically ordered at low temperatures. Here, we restricted ourselves to the low angle part of the neutron diffraction diagrams. Results are shown in Fig. 4. Inspection of these results shows, that when going from 8.0 to $1.5 \mathrm{~K}$, there is virtually no other change than some background enhancement in the low $2 \theta$ region, possibly indicating some short-range order effects. Comparison of the data of Fig. 5 with those shown in Figs. 3 and 4 makes it clear that there is no significant change when going from $295 \mathrm{~K}$ to the cryogenic temperatures. There is no visible change in the intensity of the first few lines, nor are there any

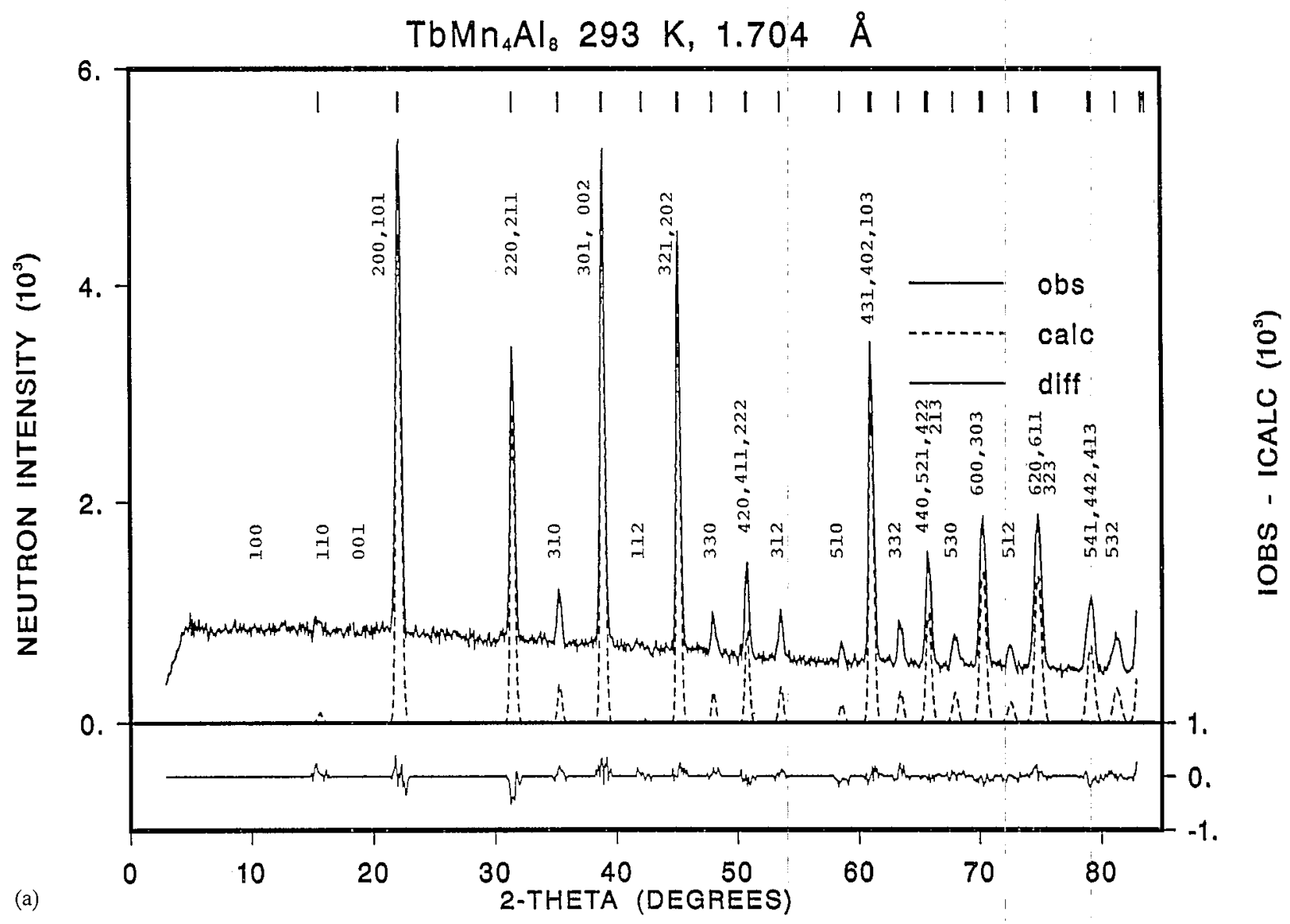

Fig. 3. Observed and calculated neutron diffraction intensities of $\mathrm{TbMn}_{4} \mathrm{Al}_{8}$ at $295 \mathrm{~K}$ (a) and $26 \mathrm{~K}$ (b). The difference between observed and calculated intensities is shown at the bottom of each figure. 


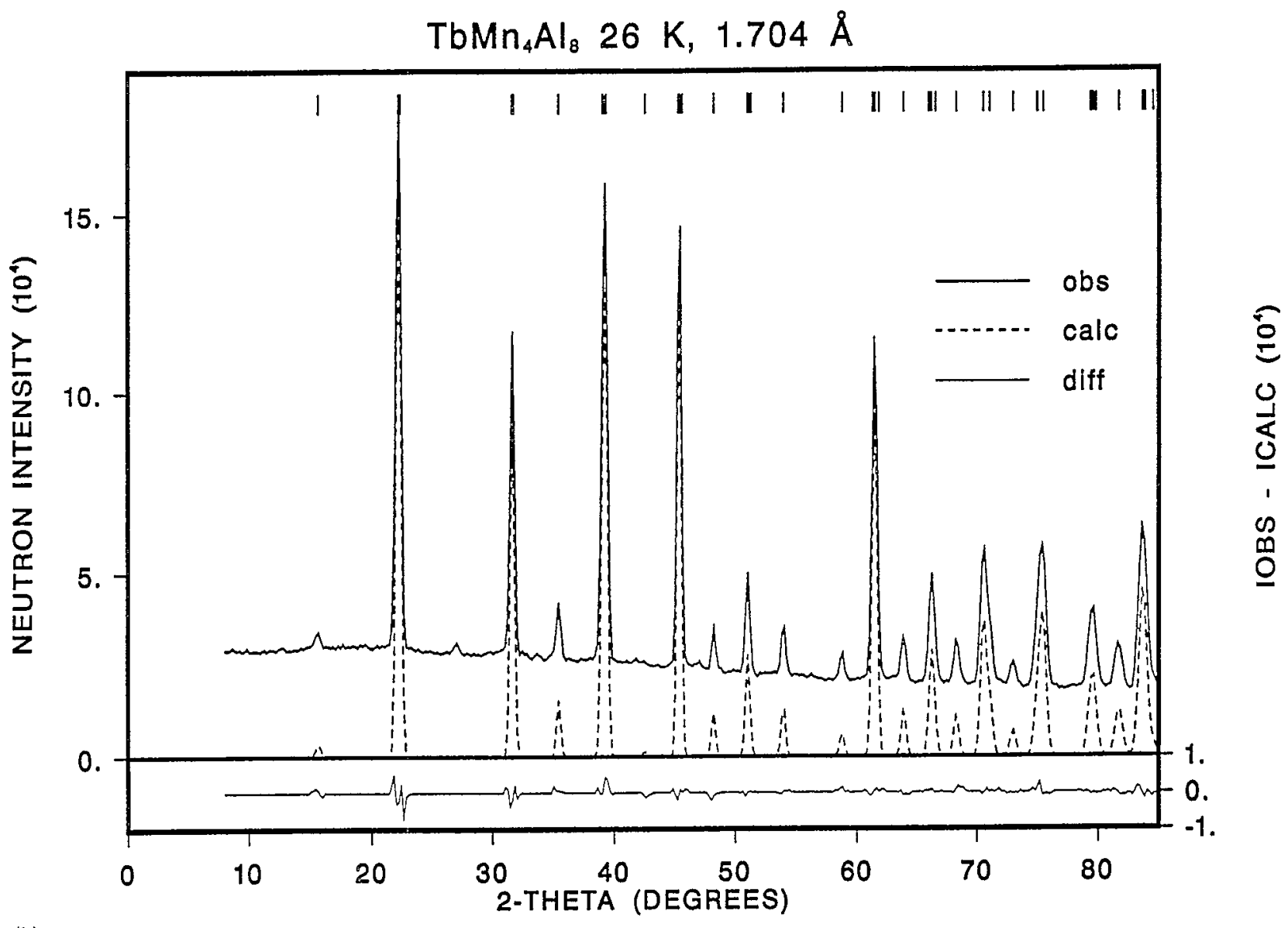

(b)

Fig. 3 (continued)

new lines of magnetic origin that would have indicated some type of antiferromagnetic ordering. These results are in agreement with the results of the magnetic measurements (Figs. 1 and 2) that have refuted the occurrence of magnetic ordering above $4.2 \mathrm{~K}$. The neutron diffraction results point to the absence of magnetic ordering even down to $1.5 \mathrm{~K}$.

\section{Concluding remarks}

In a previous investigation of the magnetic properties of the compound $\mathrm{GdMn}_{4} \mathrm{Al}_{8}$, it was found from susceptibility measurements that magnetic ordering is absent above $4.2 \mathrm{~K}$ [2]. The same conclusion can be reached from results of ${ }^{155} \mathrm{Gd}$ Mössbauer spectroscopy made on this compound, showing that hyperfine splitting at the $\mathrm{Gd}$ site is absent at $4.2 \mathrm{~K}$ [12]. Owing to the fact that Gd has the highest spin moment of all the rare-earth elements, the $\mathrm{Gd}$ compounds generally have the highest magnetic ordering temperature within a given series of isostructural rare-earth compounds. It therefore seems plausible that magnetic ordering is absent also in $\mathrm{TbMn}_{4} \mathrm{Al}_{8}$ as derived from the magnetic measurements and neutron diffraction described in the present study.

The results obtained in the course of the present investigation form a strong contrast with the results obtained for $\mathrm{TbMn}_{4} \mathrm{Al}_{8}$ by Felner and Nowick [3]. These authors reported a magnetic ordering temperature of $21 \mathrm{~K}$ for this compound together with an effective moment that deviates quite markedly from the free-ion value of $\mathrm{Tb}$.

A possible explanation for this discrepancy regarding the magnetic properties observed for $\mathrm{TbMn}_{4} \mathrm{Al}_{8}$ may be found in a difference in the degree of preferential atomic ordering of the $\mathrm{Mn}$ and $\mathrm{Al}$ atoms at the $8(\mathrm{f})(\mathrm{Mn}), 8(\mathrm{i})(\mathrm{Al})$ and $8(\mathrm{j})(\mathrm{Al})$ sites. Coldea and coworkers $[5,6]$ have proposed that localised Mn moments occur only if the $\mathrm{Mn}$ atoms occupy the 8(i) or $8(\mathrm{j})$ sites commonly preferentially occupied by $\mathrm{Al}$ atoms. In other words, localised Mn moments are only expected if there is some deviation from the ideal site occupancies of the $\mathrm{Mn}$ and $\mathrm{Al}$ atoms. If this deviation is small the number of localised moments per formula unit will also be small, and because of the correspondingly high magnetic dilution no magnetic ordering will occur. With increasing site disorder the number of localised Mn moments will increase and, for a 
$\mathrm{TbMn}_{4} \mathrm{Al}_{8} 10 \mathrm{~K}, 1.704 \AA$

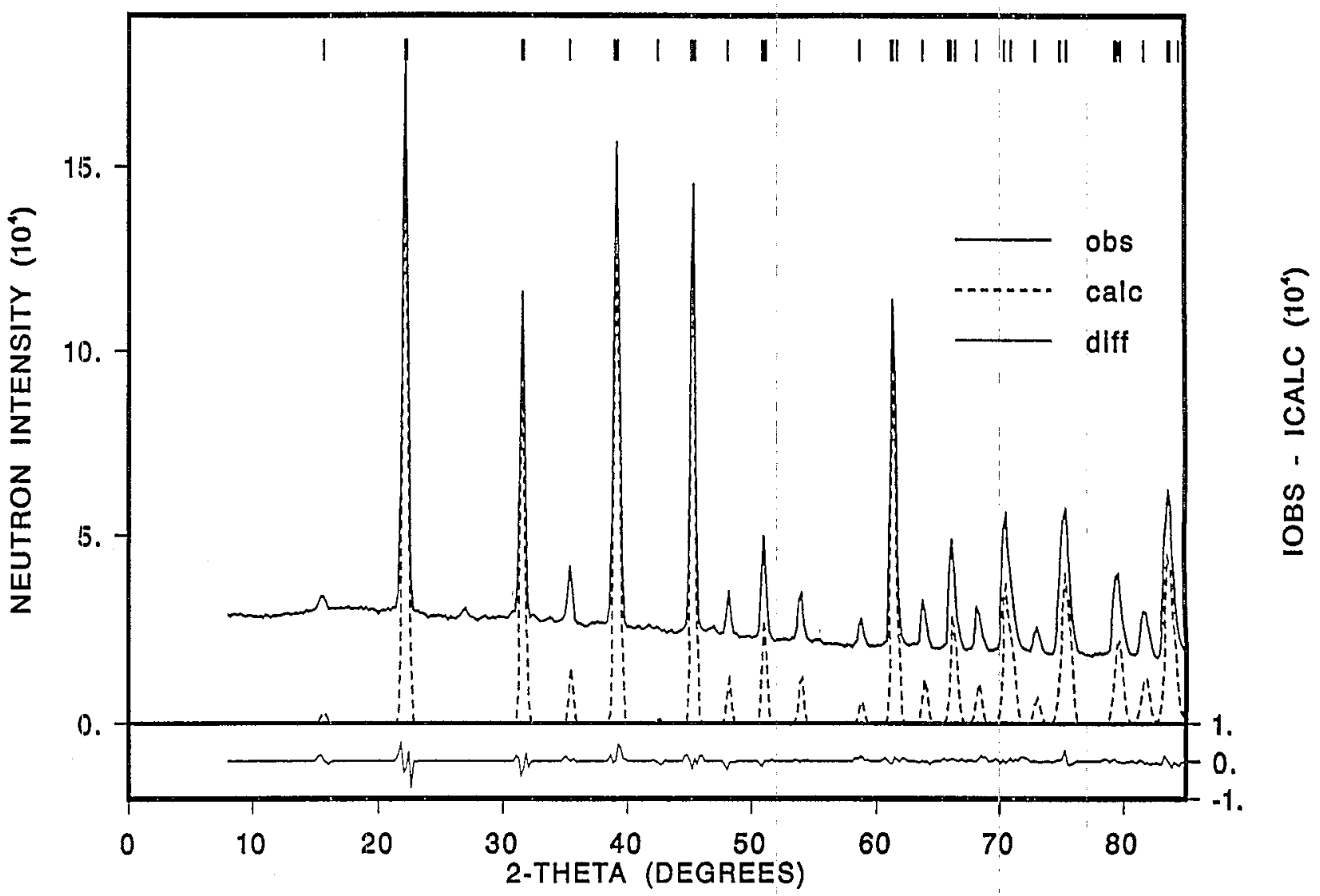

Fig. 4. Observed and calculated neutron diffraction patterns of $\mathrm{TbMn}_{4} \mathrm{Al}_{8}$ at $10 \mathrm{~K}$.

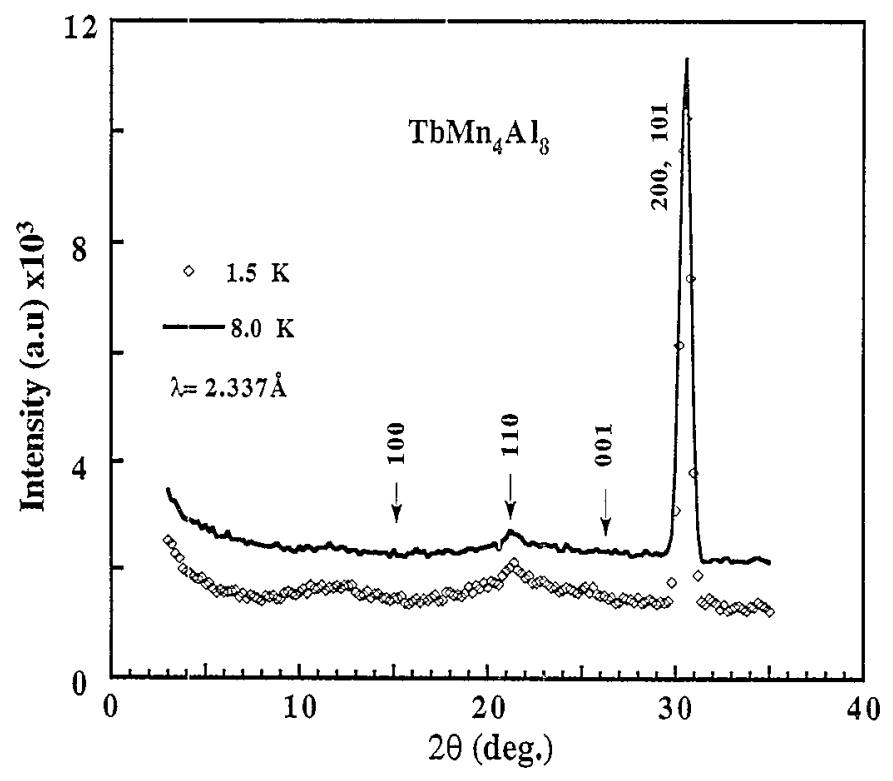

Fig. 5. Low angle part of the neutron diffraction diagram of $\mathrm{TbMn}_{4} \mathrm{Al}_{8}$ taken at $8.0 \mathrm{~K}$ (upper) and at $1.5 \mathrm{~K}$ (lower part).

sufficiently large localised-moment concentration, magnetic ordering will become manifest in the temperature range considered. The presence of the local- ised rare-earth moments will enhance this magnetic ordering and at the same time these moments will participate in the magnetic ordering process.

Our conclusion, therefore, is that the magnetic properties of the $\mathrm{RMn}_{4} \mathrm{Al}_{8}$ compounds are strongly dependent on the preparatory conditions and the concomitant degree of atomic site disorder of the $\mathrm{Mn}$ and $\mathrm{Al}$ atoms. Evidently, in the sample of $\mathrm{TbMn}_{4} \mathrm{Al}_{8}$ studied by us, this atomic site disorder is comparatively low and does not generate a number of localised $\mathrm{Mn}$ moments sufficient for magnetic ordering. At the same time, our results show that the magnetic interaction between the rare-earth moments in the $\mathrm{RMn}_{4} \mathrm{Al}_{8}$ compounds is surprisingly low, as it does not lead to magnetic ordering of the rare-earth sublattice above $1.5 \mathrm{~K}$ in $\mathrm{TbMn}_{4} \mathrm{Al}_{8}$ and above $4.2 \mathrm{~K}$ in $\mathrm{GdMn}_{4} \mathrm{Al}_{8}$ $[2,12]$.

\section{References}

[1] K.H.J. Buschow, J.H.N. van Vucht and W.W. van den Hoogenhof, J. Less-Comm, Met., 50 (1975) 145.

[2] K.H.J. Buschow and A.M. van der Kraan, J. Phys. F; 8 (1978) 921. 
[3] I. Felner and J. Nowick, J. Phys. Chem. Solids, 40 (1979) 1035.

[4] O. Moze, R.M. Ibberson, R. Caciuffo and K.H.J. Buschow, J. Less-Comm. Met., 166 (1990) 329.

[5] M. Coldea, R. Coldea and G. Borodi, IEEE Trans. Magn., Vol. 30, p. 855.

[6] R. Coldea, M. Coldea and I. Pop, IEEE Trans. Magn., Vol. 30, p. 852.

[7] J. Schefer, P. Fischer, H. Heer, A. Isacson, M. Koch and R. Thut, Nucl. Instrum. Methods A, 288 (1990) 477.
[8] H.M. Rietveld, J. Appl. Cryst., 2 (1967) 65.

[9] A.W. Hewatt, Harwell Rep. AERE R 7350, 1973.

[10] V. Sears, in K. Skoeld and D.L. Price (eds.), Methods of Experimental Physics, Vol. 23A, Academic Press, New York, 1986, p. 521.

[11] W. Schäfer, G. Will, G.M. Kalvius and J. Gal, Physica B, 156-157 (1989) 751.

[12] M.W. Dirken, Thesis, University of Leiden, 1991 (unpublished). 